\title{
RNA buffers the phase separation behavior of prion-like RNA binding proteins
}

\author{
Shovamayee Maharana, ${ }^{1}$ Jie Wang, ${ }^{1 *}$ Dimitrios K. Papadopoulos, ${ }^{1,2 *}$ Doris Richter, ${ }^{1}$ Andrey Pozniakovsky, ${ }^{1}$ Ina Poser, ${ }^{1}$ Marc Bickle, ${ }^{1}$ Sandra \\ Rizk,, ${ }^{1,3}$ Jordina Guillén-Boixet, ${ }^{1}$ Titus Franzmann, ${ }^{1}$ Marcus Jahnel, ${ }^{1}$ Lara Marrone, ${ }^{4}$ Young-Tae Chang, ${ }^{5,6}$ Jared Sterneckert, ${ }^{4}$ Pavel \\ Tomancak, ${ }^{1}$ Anthony A. Hyman, ${ }^{1} \uparrow$ Simon Alberti ${ }^{1} \uparrow$ \\ ${ }^{1}$ Max Planck Institute for Cell and Molecular Biology, Pfotenhauerstraße 108, 01307 Dresden, Germany. ${ }^{2}$ MRC Human Genetic Unit, Institute of Genetics and Molecular \\ Medicine, University of Edinburgh, Crewe Road, Edinburgh EH4 2XU, UK. ${ }^{3}$ B Cube-Center for Molecular Bioengineering, Technische Universität, Arnoldstraße 18, 01307 \\ Dresden, Germany. ${ }^{4}$ Technische Universität Dresden-Center for Molecular and Cellular Bioengineering (CMCB), DFG-Center for Regenerative Therapies Dresden, 01307 \\ Dresden, Germany. ${ }^{5}$ Center for Self-assembly and Complexity, Institute for Basic Science (IBS), Pohang 37673, Republic of Korea. ${ }^{6}$ Department of Chemistry, Pohang \\ University of Science and Technology (POSTECH), Pohang 37673, Republic of Korea.
}

*These authors contributed equally to this work.

†Corresponding author. Email: alberti@mpi-cbg.de (S.A.); hyman@mpi-cbg.de (A.A.H.)

Prion-like RNA binding proteins (RBPs) such as TDP-43 or FUS are largely soluble in the nucleus, but form solid pathological aggregates when mislocalized to the cytoplasm. What keeps these proteins soluble in the nucleus and promotes aggregation in the cytoplasm is still unknown. We report here that RNA critically regulates the phase behavior of prion-like RBPs. Low RNA/protein ratios promote phase separation into liquid droplets, whereas high ratios prevent droplet formation in vitro. Reduction of nuclear RNA levels or genetic ablation of RNA binding causes excessive phase separation and the formation of cytotoxic solid-like assemblies in cells. We propose that the nucleus is a buffered system in which high RNA concentrations keep RBPs soluble. Changes in RNA levels or RNA binding abilities of RBPs cause aberrant phase transitions.

The intracellular environment is organized into membraneless compartments, which have been termed biomolecular condensates because they form by liquid-liquid phase separation $(1,2)$. These condensates often contain RNA binding proteins (RBPs) with distinctive domains, so-called prion-like domains, which are structurally disordered and contain polar amino acids (3) (Fig. 1A). Interactions between prion-like domains and additional interactions between RNAs and RNA binding domains drive the assembly of prion-like RBPs by phase separation $(4,5)$. However, several prion-like RBPs such as FUS, TDP43, and hnRNPA1 can also undergo an aberrant transition from a liquid-like state into solid aggregates that has been linked to neurodegenerative diseases such as amyotrophic lateral sclerosis (ALS) (4-6). One important aspect of these diseases is that aggregate formation is strongly associated with their subcellular location. Aggregates in patient neurons are usually found in the cytoplasm, whereas the nucleus is devoid of the aggregating protein (7-10), although there are some noteworthy exceptions (11). The importance of cytoplasmic localization is also highlighted by the fact that disease-causing mutations frequently affect the nuclear partitioning of prion-like RBPs $(12,13)$. Protein mislocalization to the cytoplasm causes loss-of-function and gain-of-function phenotypes that are thought to underlie disease (14-17). Importantly, genetic re-localization of FUS to the nucleus in yeast strongly decreased its toxicity (18). This suggests that localization of FUS to the nuclear environment suppresses its pathological behavior. This raises two important questions: What prevents prion-like RBPs from forming solid-like aggregates in the nucleus? And why do these RBPs form aggregates in the cytoplasm?

To provide answers for these questions, we investigated the phase behavior of several prion-like RBPs (Fig. 1A). First, we determined the nuclear concentration of these proteins. The values ranged from $0.2 \mu \mathrm{M}$ for TAF-15 to $42.3 \mu \mathrm{M}$ for hnRNPA1 (Fig. 1, B to D, and supplementary methods). Next, we purified these proteins as GFP fusions and added them to a physiological buffer. At a concentration similar to the nuclear concentration $(7.6 \mu \mathrm{M})$, FUS phase separated into droplets (Fig. 1, E and F). This is in contrast to living cells, were only $1 \%$ of the nuclear FUS protein was contained in condensates (Fig. 1F), which are paraspeckles (19). The remaining $99 \%$ of nuclear FUS protein was diffusely localized. Similar observations were made for TDP43, EWSR1, TAF15, and hnRNPA1 (Fig. 1G, lower panel). This suggests that although the protein concentration is high enough for phase separation in the nucleus, there is an additional nuclear factor that prevents phase separation.

We hypothesized that nuclear RNA could regulate the 
phase behavior of prion-like RBPs. To test this idea, we performed an in vitro phase separation assay with FUS in the presence of total RNA (Fig. 2A). In agreement with previous work (20-22), we found that low amounts of RNA promoted liquid droplet formation (Fig. 2B and fig. S1, A to D). RNAcontaining droplets contained a higher FUS concentration than RNA-free droplets and they appeared slightly more viscous (fig. S2, A to C). Remarkably, however, upon further increasing the $\mathrm{RNA}$ /protein concentration ratio, the droplets became smaller and finally dissolved (Fig. 2, A and B, and fig. S3). Addition of RNase A resulted in droplet reappearance (Fig. 2D and figs. S4A, panels on the right, and S5), indicating that droplet solubilization depends on intact RNA. Similar results were obtained for EWSR1, TAF15, hnRNPA1, and TDP43 (Fig. 2C). Thus, we conclude that high RNA/ protein ratios prevent phase separation, whereas low ratios promote phase separation.

We next tested whether different types of RNAs differ with respect to their ability to dissolve FUS droplets. Individually, rRNA, tRNA or a non-coding RNA that is known to bind to FUS (Neat1) were all able to solubilize FUS droplets, suggesting a general effect, but smaller RNAs were more potent than larger ones (fig. S4, A to D). Secondary structure was important for enriching FUS in droplets, consistent with previous work (20), but both secondary structure (fig. S4, A to E) and binding affinity (fig. S6) only slightly affected droplet solubilization. We next asked if the cellular RNA concentration is high enough to suppress phase separation of FUS. We estimated that the nuclear RNA concentration is about 10.6-fold higher than required for droplet dissolution in vitro (fig. S7 and supplementary methods). However, about $1 \%$ of nuclear FUS formed condensates (Fig. 1E), called paraspeckles, by binding to the non-coding RNA Neatl (19). To test whether Neat1 could nucleate FUS droplets in the presence of a high background concentration of RNA, we added Neat1 RNA to a FUS sample that had been solubilized with tRNA. Remarkably, this led to a reappearance of FUS droplets (Fig. 2E and fig. S4F). We attribute this to the ability of Neat1 to form large RNA assemblies (fig. S4C), which subsequently recruit FUS. This suggests that highly structured RNAs such as Neat1 could act as scaffolds that promote the nucleation of condensates in the high RNA concentration environment of the nucleus. A similar scenario may apply for stress granules in the cytoplasm, which contain high amounts of structured polyAmRNA (fig. S8).

To test experimentally whether the high nuclear RNA concentration keeps FUS soluble, we microinjected RNase A into the nucleus of HeLa cells. Immediately after RNase A injection, FUS-GFP condensed into many liquid-like droplets (Fig. 3A, fig. S9, and movie S1), and this effect was not due to a general loss of nuclear integrity (figs. S10 and S11). As an al- ternative approach to decrease $\mathrm{RNA} /$ protein ratio, we injected purified FUS-GFP into the nucleus, which led to an immediate increase in the number and size of nuclear FUS assemblies (fig. S12). RNase A microinjection into the nucleus also triggered rapid phase separation of hnRNPA1, EWSR1, TDP43 and TAF15 (figs. S13 and S14). To investigate whether FUS forms complexes with RNA in living cells, we employed fluorescence correlation spectroscopy (FCS). We identified two populations of FUS, one slow moving and one fast moving (see supplementary methods for details). We estimate that the amount of slow FUS in the nucleus is 10-fold greater than in the cytoplasm (Fig. 3, B to D; fig. S15, A to E; and supplementary methods). The fraction of slow FUS in the nucleus was decreased by mutating RNA binding domains in FUS (FUS-mutRRM/ZnF, FUS-mutRGG2) and was further decreased by removing all RNA binding domains (FUS-PLD) (Fig. 3, E and F, and figs. S15, F to I; S16; and S17). This indicates that a large fraction of nuclear FUS is complexed with RNA. To further investigate the solubilizing role of RNA, we performed genetic experiments with transfected FUS-GFP encoding plasmids. We observed that the number of nuclear FUS assemblies was directly proportional to the nuclear FUS concentration (Fig. 3G). We further found that FUS variants with a weaker capacity to bind RNA generally formed a higher number of assemblies (Fig. 3, H to J, and figs. S16 and S17). Thus, reduced RNA binding directly affects the solubility and decreases the saturation concentration at which FUS phase separates.

We showed previously that FUS in vitro initially forms liquid-like assemblies, but these mature into more solid-like gels and fibrils over time (5). These solid-like states are reminiscent of pathological aggregates in $\operatorname{ALS}(8,9)$. Thus, we next tested whether addition of RNA prevents the formation of fibrils in vitro. Remarkably, addition of RNA kept the droplets in a soluble state, and fibers were not seen (Fig. 4A). We next investigated whether RNA also changes the material properties of FUS assemblies in vivo. We set up an in vivo aging assay, in which we microinjected RNase A into HeLa cells and then followed the dynamics of the liquid-like drops. After about $30 \mathrm{~min}$, the FUS drops no longer fused (Fig. 4, B and C, and movie S2), but stuck together in large clusters, similar to phenotypes seen previously in vitro (Fig. 4D and movie S3). A change in the material properties was also evident from photobleaching experiments (Fig. 4, E and F, and fig. S18, A and B). Similar results were obtained for TDP43, but the transition was much faster (fig. S19 and movie S4). We next used a genetic approach to test how RNA binding affects the dynamics of FUS in vivo. Complete abrogation of RNA binding resulted in a dramatic decrease of mobile FUS (Fig. 4, G, H, and J, and fig. S18C) and the formation of sticky droplet clusters (Fig. 4K). Lastly, we employed a chemical approach using the dye F22 to reduce RNA binding (23). In F22-treated cells, 
the fraction of RNA-bound FUS was strongly diminished (fig. S20 and supplementary methods) and this caused a strong reduction in the mobile fraction of FUS (Fig. 4, I and J, and fig. S18, E and F). Together, these findings show that RNA keeps condensates formed by prion-like RBPs in a dynamic state and prevents the formation of solid assemblies that could cause disease.

To investigate how reduced RNA binding affects cell viability, we transiently transfected HeLa cells with wild type and mutant FUS and monitored cell survival. Expressing a nuclear variant with reduced RNA binding (FUSmutRRM/ZnF) affected the rate of cell death only slightly (Fig. 4, L and M, and fig. S16), presumably because the high nuclear RNA concentration compensates for the genetic defect. However, targeting the very same variant to the cytoplasm by removing the NLS (FUS-mutRRM/ZnF $\Delta$ NLS) led to a strong increase in cell death, which was likely caused by the high propensity of this variant to form solid aggregates (figs. S21 to S23). Importantly, this increase was not observed for a cytosolic variant of FUS with normal RNA binding (FUS $\triangle \mathrm{NLS})$. Thus, we conclude that excessive phase separation in the cytoplasm due to low RNA levels induces a pathological state that leads to cell death.

One of the key questions in protein misfolding diseases caused by prion-like RBPs is why these proteins aggregate in the cytoplasm, rather than the nucleus. In this paper, we show that this is due in part to different RNA concentrations in the cytoplasm and the nucleus. More specifically, the higher RNA concentration in the nucleus suppresses phase separation of prion-like RBPs, while the lower concentration in the cytoplasm stimulates phase separation. Therefore, by keeping the proteins in the nucleus, the cell ensures that they are in a soluble and non-toxic state, shuttling them out of the nucleus only upon stress. After removal of stress, the proteins shuttle back into the nucleus where they are again kept in a soluble and well-mixed state. The consequence is that any insult that prolongs the stress will tend to increase the propensity for aggregation because they prolong the time these proteins spend in the cytoplasm (fig. S24).

Our data also has important implications for the control of phase separation in cells. We find that paraspeckles are likely induced by locally concentrating Neat1 RNA, which has a strong affinity for FUS. Similar phenomena have been seen for nucleoli, who depend on local production of ribosomal RNA (24). Therefore, at least in the nucleus, local production of RNAs with high affinity for specific RBPs could provide the specificity to induce phase separation in a system buffered by non-specifically interacting RNA. Thus, the phase behavior of FUS in the nucleus is likely controlled by many different types of specific and nonspecific RNAs. This situation does not apply to the cytoplasm. Here, the RNA concentration is only slightly higher than the concentration required to suppress phase separation in vitro, and there is no buffering of phase separation by RNA. This results in a much higher propensity of FUS to phase separate. However, it also brings with it an increase in the tendency of FUS to form cytotoxic solidlike aggregates. Indeed, high amounts of RNA have been shown to suppress the toxicity of prion-like RBPs (25-28). Moreover, there are many cases of familial ALS in which mutated prion-like RBPs mislocalize to the cytoplasm and form cytotoxic aggregates. For example, mutations in FUS have been shown to increase its cytoplasmic concentration, thus causing the formation of aberrant solid-like aggregates $(8,9$, 29-31). We predict that local changes in RNA levels or RNA binding abilities of proteins are frequent causes of age-related protein misfolding diseases.

\section{REFERENCES AND NOTES}

1. S. F. Banani, H. O. Lee, A. A. Hyman, M. K. Rosen, Biomolecular condensates: Organizers of cellular biochemistry. Nat. Rev. Mol. Cell Biol. 18, 285-298 (2017). doi:10.1038/nrm.2017.7 Medline

2. Y. Shin, C. P. Brangwynne, Liquid phase condensation in cell physiology and disease. Science 357, eaaf4382 (2017). doi:10.1126/science.aaf4382 Medline

3. Z. M. March, O. D. King, J. Shorter, Prion-like domains as epigenetic regulators, scaffolds for subcellular organization, and drivers of neurodegenerative disease. Brain Res. 1647, 9-18 (2016). doi:10.1016/j.brainres.2016.02.037

4. A. Patel, H. O. Lee, L. Jawerth, S. Maharana, M. Jahnel, M. Y. Hein, S. Stoynov, J. Mahamid, S. Saha, T. M. Franzmann, A. Pozniakovski, I. Poser, N. Maghelli, L. A. Royer, M. Weigert, E. W. Myers, S. Grill, D. Drechsel, A. A. Hyman, S. Alberti, A Liquid-to-solid phase transition of the ALS protein FUS accelerated by disease mutation. Cell 162, 1066-1077 (2015). doi:10.1016/j.cell.2015.07.047 Medline

5. A. Molliex, J. Temirov, J. Lee, M. Coughlin, A. P. Kanagaraj, H. J. Kim, T. Mittag, J. P. Taylor, Phase separation by low complexity domains promotes stress granule assembly and drives pathological fibrillization. Cell 163, 123-133 (2015). doi:10.1016/i.cell.2015.09.015 Medline

6. D. Mateju, T. M. Franzmann, A. Patel, A. Kopach, E. E. Boczek, S. Maharana, H. O. Lee, S. Carra, A. A. Hyman, S. Alberti, An aberrant phase transition of stress granules triggered by misfolded protein and prevented by chaperone function. EMBO J. 36, 1669-1687 (2017). doi:10.15252/embj.201695957 Medline

7. J. P. Taylor, R. H. Brown Jr., D. W. Cleveland, Decoding ALS: From genes to mechanism. Nature 539, 197-206 (2016). doi:10.1038/nature20413 Medline

8. C. Vance, B. Rogelj, T. Hortobágyi, K. J. De Vos, A. L. Nishimura, J. Sreedharan, X. Hu, B. Smith, D. Ruddy, P. Wright, J. Ganesalingam, K. L. Williams, V. Tripathi, S. Al-Saraj, A. Al-Chalabi, P. N. Leigh, I. P. Blair, G. Nicholson, J. de Belleroche, J.-M. Gallo, C. C. Miller, C. E. Shaw, Mutations in FUS, an RNA processing protein, cause familial amyotrophic lateral sclerosis type 6. Science 323, 1208-1211 (2009). doi:10.1126/science. 1165942 Medline

9. T. J. Kwiatkowski Jr., D. A. Bosco, A. L. Leclerc, E. Tamrazian, C. R. Vanderburg, C. Russ, A. Davis, J. Gilchrist, E. J. Kasarskis, T. Munsat, P. Valdmanis, G. A. Rouleau, B. A. Hosler, P. Cortelli, P. J. de Jong, Y. Yoshinaga, J. L. Haines, M. A. PericakVance, J. Yan, N. Ticozzi, T. Siddique, D. McKenna-Yasek, P. C. Sapp, H. R. Horvitz, J. E. Landers, R. H. Brown Jr., Mutations in the FUS/TLS gene on chromosome 16 cause familial amyotrophic lateral sclerosis. Science 323, 1205-1208 (2009). doi:10.1126/science. 1166066 Medline

10. M. Neumann, D. M. Sampathu, L. K. Kwong, A. C. Truax, M. C. Micsenyi, T. T. Chou, J. Bruce, T. Schuck, M. Grossman, C. M. Clark, L. F. McCluskey, B. L. Miller, E. Masliah, I. R. Mackenzie, H. Feldman, W. Feiden, H. A. Kretzschmar, J. Q. Trojanowski, V. M.-Y. Lee, Ubiquitinated TDP-43 in frontotemporal lobar degeneration and amyotrophic lateral sclerosis. Science 314, 130-133 (2006). doi:10.1126/science. 1134108 Medline

11. M. Neumann, L. K. Kwong, E. B. Lee, E. Kremmer, A. Flatley, Y. Xu, M. S. Forman, D. Troost, H. A. Kretzschmar, J. Q. Trojanowski, V. M.-Y. Lee, Phosphorylation of 
S409/410 of TDP-43 is a consistent feature in all sporadic and familial forms of TDP-43 proteinopathies. Acta Neuropathol. 117, 137-149 (2009). doi:10.1007/s00401-008-0477-9 Medline

12. H. Deng, K. Gao, J. Jankovic, The role of FUS gene variants in neurodegenerative diseases. Nat. Rev. Neurol. 10, 337-348 (2014). doi:10.1038/nrneurol.2014.78 Medline

13. D. Dormann, R. Rodde, D. Edbauer, E. Bentmann, I. Fischer, A. Hruscha, M. E. Than, I. R. A. Mackenzie, A. Capell, B. Schmid, M. Neumann, C. Haass, ALS-associated fused in sarcoma (FUS) mutations disrupt Transportin-mediated nuclear import. EMBO J. 29, 2841-2857 (2010). doi:10.1038/emboj.2010.143 Medline

14. S. J. Barmada, G. Skibinski, E. Korb, E. J. Rao, J. Y. Wu, S. Finkbeiner, Cytoplasmic mislocalization of TDP-43 is toxic to neurons and enhanced by a mutation associated with familial amyotrophic lateral sclerosis. J. Neurosci. 30, 639-649 (2010). doi:10.1523/JNEUROSCI.4988-09.2010 Medline

15. D. C. Diaper, Y. Adachi, B. Sutcliffe, D. M. Humphrey, C. J. H. Elliott, A. Stepto, Z. N. Ludlow, L. Vanden Broeck, P. Callaerts, B. Dermaut, A. Al-Chalabi, C. E. Shaw, I. M. Robinson, F. Hirth, Loss and gain of Drosophila TDP-43 impair synaptic efficacy and motor control leading to age-related neurodegeneration by loss-of-function phenotypes. Hum. Mol. Genet. 22, 1539-1557 (2013). doi:10.1093/hmg/ddt005 Medline

16. J. Scekic-Zahirovic, O. Sendscheid, H. El Oussini, M. Jambeau, Y. Sun, S. Mersmann, M. Wagner, S. Dieterlé, J. Sinniger, S. Dirrig-Grosch, K. Drenner, M.-C. Birling, J. Qiu, Y. Zhou, H. Li, X.-D. Fu, C. Rouaux, T. Shelkovnikova, A. Witting, A. C. Ludolph, F. Kiefer, E. Storkebaum, C. Lagier-Tourenne, L. Dupuis, Toxic gain of function from mutant FUS protein is crucial to trigger cell autonomous motor neuron loss. EMBO J. 35, 1077-1097 (2016). doi:10.15252/embj.201592559 Medline

17. A. Sharma, A. K. Lyashchenko, L. Lu, S. E. Nasrabady, M. Elmaleh, M. Mendelsohn, A. Nemes, J. C. Tapia, G. Z. Mentis, N. A. Shneider, ALS-associated mutant FUS induces selective motor neuron degeneration through toxic gain of function. Nat. Commun. 7, 10465 (2016). doi:10.1038/ncomms10465 Medline

18. Z. Sun, Z. Diaz, X. Fang, M. P. Hart, A. Chesi, J. Shorter, A. D. Gitler, Molecular determinants and genetic modifiers of aggregation and toxicity for the ALS disease protein FUS/TLS. PLOS Biol. 9, e1000614 (2011). doi:10.1371/journal.pbio.1000614 Medline

19. A. H. Fox, S. Nakagawa, T. Hirose, C. S. Bond, Paraspeckles: Where long noncoding RNA meets phase separation. Trends Biochem. Sci. 43, 124-135 (2017). doi:10.1016/i.tibs.2017.12.001

20. S. Saha, C. A. Weber, M. Nousch, O. Adame-Arana, C. Hoege, M. Y. Hein, E. Osborne-Nishimura, J. Mahamid, M. Jahnel, L. Jawerth, A. Pozniakovski, C. R. Eckmann, F. Jülicher, A. A. Hyman, Polar positioning of phase-separated liquid compartments in cells regulated by an mRNA competition mechanism. Cell 166, 1572-1584.e16 (2016). doi:10.1016/j.cell.2016.08.006

21. S. Elbaum-Garfinkle, Y. Kim, K. Szczepaniak, C. C.-H. Chen, C. R. Eckmann, S. Myong, C. P. Brangwynne, The disordered P granule protein LAF-1 drives phase separation into droplets with tunable viscosity and dynamics. Proc. Natl. Acad. Sci. U.S.A. 112, 7189-7194 (2015). doi:10.1073/pnas.1504822112 Medline

22. H. Zhang, S. Elbaum-Garfinkle, E. M. Langdon, N. Taylor, P. Occhipinti, A. A. Bridges, C. P. Brangwynne, A. S. Gladfelter, RNA controls polyQ protein phase transitions. Mol. Cell 60, 220-230 (2015). doi:10.1016/j.molcel.2015.09.017 Medline

23. Q. Li, Y. Kim, J. Namm, A. Kulkarni, G. R. Rosania, Y.-H. Ahn, Y.-T. Chang, RNAselective, live cell imaging probes for studying nuclear structure and function. Chem. Biol. 13, 615-623 (2006). doi:10.1016/j.chembiol.2006.04.007 Medline

24. J. Berry, S. C. Weber, N. Vaidya, M. Haataja, C. P. Brangwynne, RNA transcription modulates phase transition-driven nuclear body assembly. Proc. Natl. Acad. Sci. U.S.A. 112, E5237-E5245 (2015). doi:10.1073/pnas.1509317112 Medline

25. A. Kitamura, Y. Nakayama, A. Shibasaki, A. Taki, S. Yuno, K. Takeda, M. Yahara, N. Tanabe, M. Kinjo, Interaction of RNA with a C-terminal fragment of the amyotrophic lateral sclerosis-associated TDP43 reduces cytotoxicity. Sci. Rep. 6 , 19230 (2016). doi:10.1038/srep19230 Medline

26. T. Ishiguro, N. Sato, M. Ueyama, N. Fujikake, C. Sellier, A. Kanegami, E. Tokuda, B. Zamiri, T. Gall-Duncan, M. Mirceta, Y. Furukawa, T. Yokota, K. Wada, J. P. Taylor, C. E. Pearson, N. Charlet-Berguerand, H. Mizusawa, Y. Nagai, K. Ishikawa, Regulatory role of RNA chaperone TDP-43 for RNA misfolding and repeat- associated translation in SCA31. Neuron 94, 108-124.e7 (2017). doi:10.1016/j.neuron.2017.02.046 Medline

27. Y.-C. Huang, K.-F. Lin, R.-Y. He, P.-H. Tu, J. Koubek, Y.-C. Hsu, J. J.-T. Huang, Inhibition of TDP-43 aggregation by nucleic acid binding. PLOS ONE 8, e64002 (2013). doi:10.1371/journal.pone.0064002 Medline

28. Y. M. Ayala, P. Zago, A. D’Ambrogio, Y.-F. Xu, L. Petrucelli, E. Buratti, F. E. Baralle, Structural determinants of the cellular localization and shuttling of TDP-43. J. Cell Sci. 121, 3778-3785 (2008). doi:10.1242/jcs.038950 Medline

29. M. Sabatelli, A. Moncada, A. Conte, S. Lattante, G. Marangi, M. Luigetti, M. Lucchini, M. Mirabella, A. Romano, A. Del Grande, G. Bisogni, P. N. Doronzio, P. M. Rossini, M. Zollino, Mutations in the 3' untranslated region of FUS causing FUS overexpression are associated with amyotrophic lateral sclerosis. Hum. Mol. Genet. 22, 4748-4755 (2013). doi:10.1093/hmg/ddt328 Medline

30. S. Dini Modigliani, M. Morlando, L. Errichelli, M. Sabatelli, I. Bozzoni, An ALSassociated mutation in the FUS $3^{\prime}$-UTR disrupts a microRNA-FUS regulatory circuitry. Nat. Commun. 5, 4335 (2014). doi:10.1038/ncomms5335 Medline

31. Y. Shang, E. J. Huang, Mechanisms of FUS mutations in familial amyotrophic lateral sclerosis. Brain Res. 1647, 65-78 (2016). doi:10.1016/i.brainres.2016.03.036

32. I. Poser, M. Sarov, J. R. A. Hutchins, J.-K. Hériché, Y. Toyoda, A. Pozniakovsky, D. Weigl, A. Nitzsche, B. Hegemann, A. W. Bird, L. Pelletier, R. Kittler, S. Hua, R. Naumann, M. Augsburg, M. M. Sykora, H. Hofemeister, Y. Zhang, K. Nasmyth, K. P. White, S. Dietzel, K. Mechtler, R. Durbin, A. F. Stewart, J.-M. Peters, F. Buchholz, A. A. Hyman, BAC TransgeneOmics: A high-throughput method for exploration of protein function in mammals. Nat. Methods 5, 409-415 (2008). doi:10.1038/nmeth.1199 Medline

33. B. A. Ozdilek, V. F. Thompson, N. S. Ahmed, C. I. White, R. T. Batey, J. C. Schwartz, Intrinsically disordered RGG/RG domains mediate degenerate specificity in RNA binding. Nucleic Acids Res. 45, 7984-7996 (2017). doi:10.1093/nar/gkx460 Medline

34. X. Liu, C. Niu, J. Ren, J. Zhang, X. Xie, H. Zhu, W. Feng, W. Gong, The RRM domain of human fused in sarcoma protein reveals a non-canonical nucleic acid binding site. Biochim. Biophys. Acta 1832, 375-385 (2013). doi:10.1016/i.bbadis.2012.11.012 Medline

35. J. G. Daigle, N. A. Lanson Jr., R. B. Smith, I. Casci, A. Maltare, J. Monaghan, C. D. Nichols, D. Kryndushkin, F. Shewmaker, U. B. Pandey, RNA-binding ability of FUS regulates neurodegeneration, cytoplasmic mislocalization and incorporation into stress granules associated with FUS carrying ALS-linked mutations. Hum. Mol. Genet. 22, 1193-1205 (2013). doi:10.1093/hmg/dds526 Medline

36. C. D. Nguyen, R. E. Mansfield, W. Leung, P. M. Vaz, F. E. Loughlin, R. P. Grant, J. P. Mackay, Characterization of a family of RanBP2-type zinc fingers that can recognize single-stranded RNA. J. Mol. Biol. 407, 273-283 (2011). doi:10.1016/j.jmb.2010.12.041 Medline

37. L. Marrone, I. Poser, I. Casci, J. Japtok, P. Reinhardt, A. Janosch, C. Andree, H. O. Lee, C. Moebius, E. Koerner, L. Reinhardt, M. E. Cicardi, K. Hackmann, B. Klink, A. Poletti, S. Alberti, M. Bickle, A. Hermann, U. B. Pandey, A. A. Hyman, J. L. Sterneckert, Isogenic FUS-eGFP iPSC reporter lines enable quantification of FUS stress granule pathology that is rescued by drugs inducing autophagy. Stem Cell Reports 10, 375-389 (2018). doi:10.1016/j.stemcr.2017.12.018 Medline

38. C. B. Müller, A. Loman, V. Pacheco, F. Koberling, D. Willbold, W. Richtering, J. Enderlein, Precise measurement of diffusion by multi-color dual-focus fluorescence correlation spectroscopy. Europhys. Lett. 83, 46001 (2008). doi:10.1209/0295-5075/83/46001

39. M. Y. Hein, N. C. Hubner, I. Poser, J. Cox, N. Nagaraj, Y. Toyoda, I. A. Gak, I. Weisswange, J. Mansfeld, F. Buchholz, A. A. Hyman, M. Mann, A human interactome in three quantitative dimensions organized by stoichiometries and abundances. Cell 163, 712-723 (2015). doi:10.1016/i.cell.2015.09.053 Medline

40. A. Fujioka, K. Terai, R. E. Itoh, K. Aoki, T. Nakamura, S. Kuroda, E. Nishida, M. Matsuda, Dynamics of the Ras/ERK MAPK cascade as monitored by fluorescent probes. J. Biol. Chem. 281, 8917-8926 (2006). doi:10.1074/jbc.M509344200 Medline

41. A. Mazumder, T. Roopa, A. Basu, L. Mahadevan, G. V. Shivashankar, Dynamics of chromatin decondensation reveals the structural integrity of a mechanically prestressed nucleus. Biophys. J. 95, 3028-3035 (2008). doi:10.1529/biophysj.108.132274 Medline

42. F. Tang, K. Lao, M. A. Surani, Development and applications of single-cell 
transcriptome analysis. Nat. Methods 8 (suppl.), S6-S11 (2011). doi:10.1038/nmeth.1557 Medline

43. T. von der Haar, A quantitative estimation of the global translational activity in logarithmically growing yeast cells. BMC Syst. Biol. 2, 87 (2008). doi:10.1186/1752-0509-2-87 Medline

44. J. Guillén-Boixet, V. Buzon, X. Salvatella, R. Méndez, CPEB4 is regulated during cell cycle by ERK2/Cdk1-mediated phosphorylation and its assembly into liquidlike droplets. elife 5, e19298 (2016). doi:10.7554/eLife.19298 Medline

45. C. Colombrita, E. Zennaro, C. Fallini, M. Weber, A. Sommacal, E. Buratti, V. Silani, A. Ratti, TDP-43 is recruited to stress granules in conditions of oxidative insult. J. Neurochem. 111, 1051-1061 (2009). doi:10.1111/j.1471-4159.2009.06383.x Medline

46. J. I. Hoell, E. Larsson, S. Runge, J. D. Nusbaum, S. Duggimpudi, T. A. Farazi, M. Hafner, A. Borkhardt, C. Sander, T. Tuschl, RNA targets of wild-type and mutant FET family proteins. Nat. Struct. Mol. Biol. 18, 1428-1431 (2011). doi:10.1038/nsmb.2163 Medline

47. D. L. Jarvis, in Methods in Enzymology, J. Lorsch, Ed. (Academic Press, 2014; http://www.sciencedirect.com/science/article/pii/B9780124200708000131), vol. 536 of Laboratory Methods in Enzymology: Protein Part A, pp. 149-163.

48. J. C. Schwartz, X. Wang, E. R. Podell, T. R. Cech, RNA seeds higher-order assembly of FUS protein. Cell Reports 5, 918-925 (2013). doi:10.1016/j.celrep.2013.11.017 Medline

49. C. M. Clemson, J. N. Hutchinson, S. A. Sara, A. W. Ensminger, A. H. Fox, A. Chess, J. B. Lawrence, An architectural role for a nuclear noncoding RNA: NEAT1 RNA is essential for the structure of paraspeckles. Mol. Cell 33, 717-726 (2009). doi:10.1016/j.molcel.2009.01.026 Medline

50. M. Jahnel, M. Behrndt, A. Jannasch, E. Schäffer, S. W. Grill, Measuring the complete force field of an optical trap. Opt. Lett. 36, 1260-1262 (2011). doi:10.1364/OL.36.001260 Medline

51. J. A. Ream, L. K. Lewis, K. A. Lewis, Rapid agarose gel electrophoretic mobility shift assay for quantitating protein: RNA interactions. Anal. Biochem. 511, 36-41 (2016). doi:10.1016/i.ab.2016.07.027 Medline

52. J. R. Lakowicz, Principles of Fluorescence Spectroscopy (Springer US, ed. 3, 2006: //www.springer.com/de/book/9780387312781).

\section{ACKNOWLEDGMENTS}

We thank the members of MPI-CBG for discussions; B. Borgonovo (Chromatography Facility); R. Wegner (Protein Expression and Purification Facility); A. Bogdanova for providing vectors; M. Leuschner and A. Ssykor for preparing BAC lines; J. Peychl, B. Nitzsche, and B. Schroth-Diez (Light Microscopy Facility); C. Andree and C. Möbius (Technology Development Studio); J. Jarrells (DNA microarray facility) and the FACS facility; and D. Dormann and E. Bogaert for providing reagents. Funding: We acknowledge funding from the Max Planck Society, the ERC (no. 725836 and 643417), the BMBF (01ED1601A, 031A359A), and the JPND (CureALS). S.M. was supported by a fellowship of the Humboldt Foundation (3.5-INI/1155756 STP), L.M. by the Hans und IIse Breuer Stiftung, and J.G.-B. by an EMBO fellowship (ALTF 406-2017). Author contributions: S.M. and S.A. designed and coordinated the project. J.W. performed in vitro experiments with RBPs. D.K.P. performed FCS experiments, J.G.-B. the RIP, and T.F. the RNA binding assay. D.R., A.P., and S.R. generated plasmids. I.P. generated HeLa lines. L.M. and J.S. provided iPSC-derived cell lines. M.B. analyzed the cell viability assay. M.J. performed optical tweezer experiments. Y.T.C. provided the dye F22. S.M., A.A.H., and S.A. drafted the manuscript with input from P.T. All authors contributed to data analysis and interpretation. Competing interests: The dye F22 was covered by U.S. patent US 7790896 B2 awarded to Y.-T.C. The other authors declare no competing interests. Data and materials availability: All data are available in the manuscript or the supplementary materials.

\section{SUPPLEMENTARY MATERIALS}

www.sciencemag.org/cgi/content/full/science.aar7366/DC1

Materials and Methods

Figs. S1 to S24

References (32-52)

Movies S1 to S4

12 December 2017; accepted 4 April 2018

Published online 12 April 2018

10.1126/science.aar7366 


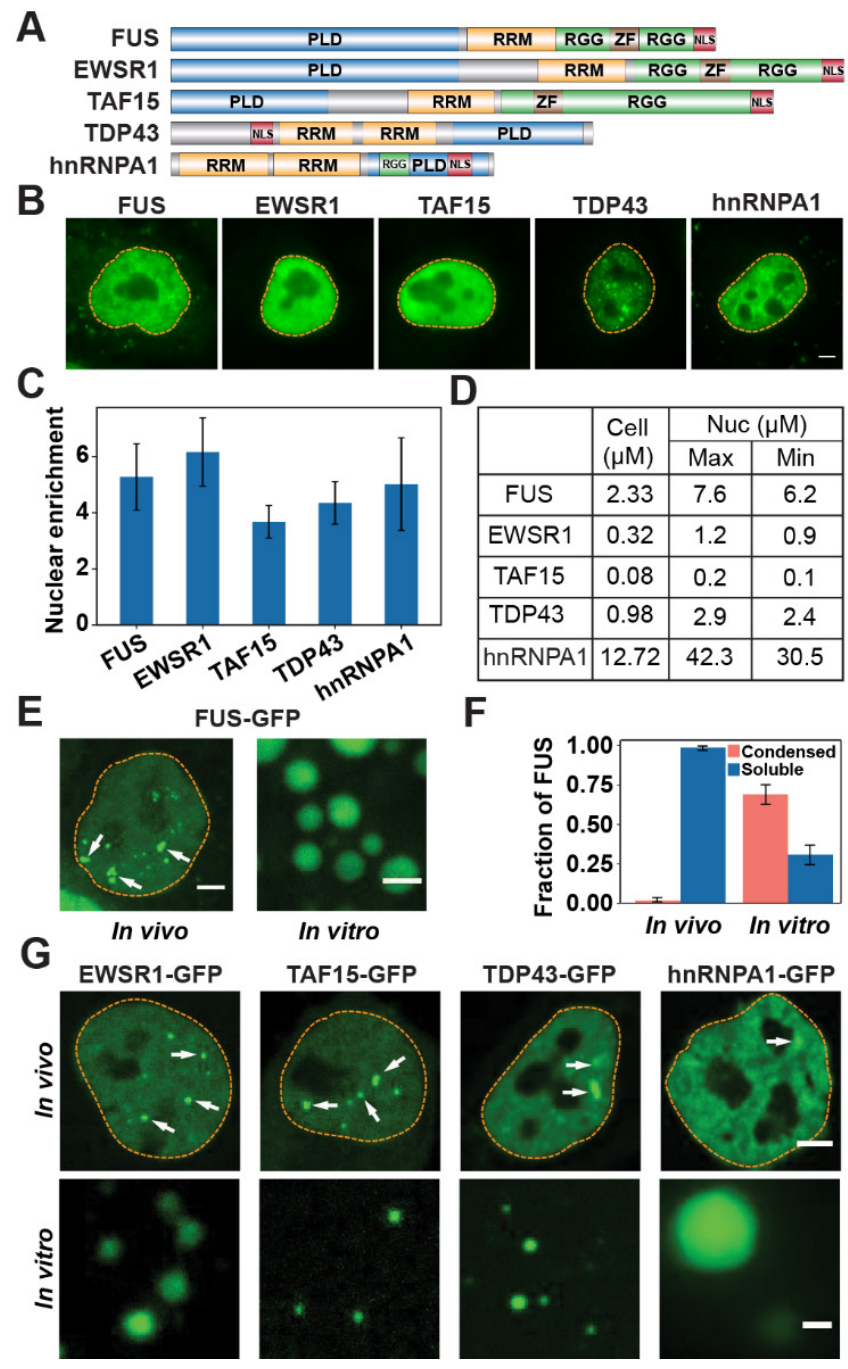

Fig. 1. Prion-like RBPs phase separate at their physiological concentrations. (A) Domain structure. PLD: prion-like domain; RRM: RNA recognition motif, RGG: arginine- and glycine-rich region; ZF: zinc finger; NLS: nuclear localization sequence. (B) Representative images of immunostained HeLa cells. Dashed line indicates nuclear boundary. (C) Quantification of the nuclear enrichment of RBPs. Error bars represent SD. Scale bar, $5 \mu \mathrm{m}$. (D) Calculated cellular and nuclear concentrations of RBPs in HeLa cells. (E) Left: Live HeLa cell nucleus expressing GFP-tagged FUS from a BAC. Arrows point to paraspeckles. Right: FUS-GFP phaseseparated in vitro at $7.5 \mu \mathrm{M}$. Scale bar, $2 \mu \mathrm{m}$. (F) Quantification of the fraction of FUS present in the condensed or the soluble state in vivo and in vitro. Error bars represent SD. (G) Top panel: HeLa cell nuclei expressing GFP-tagged RBPs from BACs. White arrows indicate condensates. Lower panel: purified RBPs phase separate at their respective nuclear concentrations. Scale bar, $2 \mu \mathrm{m}$. 
A

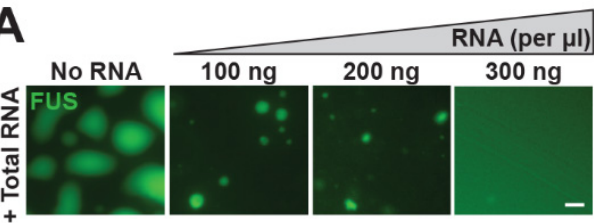

\section{B}

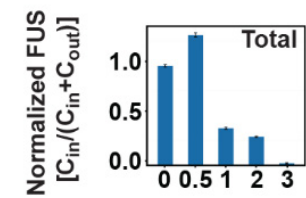

C

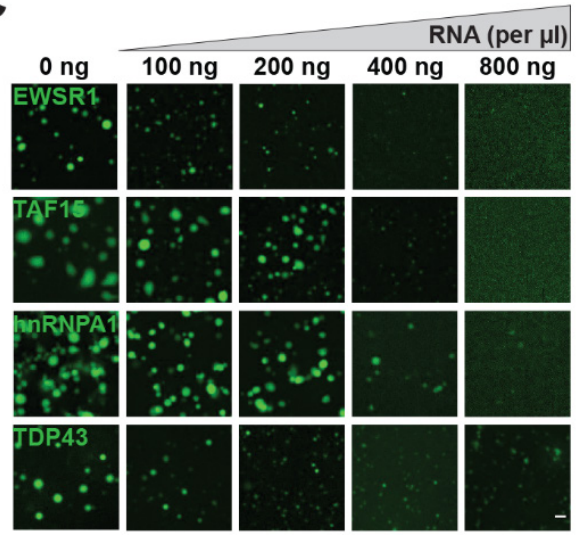

D $300 \mathrm{ng}$ Total RNA

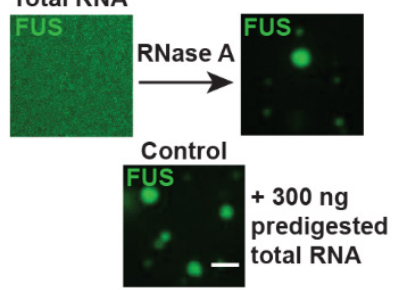

E

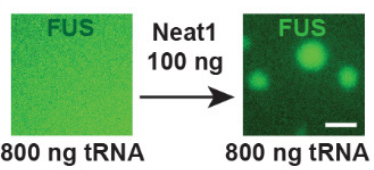

Fig. 2. RNA regulates the phase behavior of prion-like RBPs. (A) Representative images of purified FUS-GFP $(5 \mu \mathrm{M})$ in vitro in the presence of total RNA. (B) Quantification of the fraction of condensed FUS-GFP. Error bars represent SD. (C) In vitro phase separation assay with EWSR1, TAF15, hnRNPA1 or TDP43 in the presence of total RNA. (D) Addition of RNase A to a sample of FUS-GFP $(5 \mu \mathrm{M})$ solubilized with $300 \mathrm{ng} / \mu \mathrm{l}$ of total RNA. Scale bar, $2 \mu \mathrm{m}$. (E) Left: FUS-GFP $(5 \mu \mathrm{M})$ solubilized with $800 \mathrm{ng} / \mu \mathrm{l}$ tRNA in vitro. Right: Addition of $100 \mathrm{ng} / \mu \mathrm{l}$ Neat1 RNA triggers FUS phase separation in the presence of tRNA $(800 \mathrm{ng} / \mu \mathrm{l})$. 
A
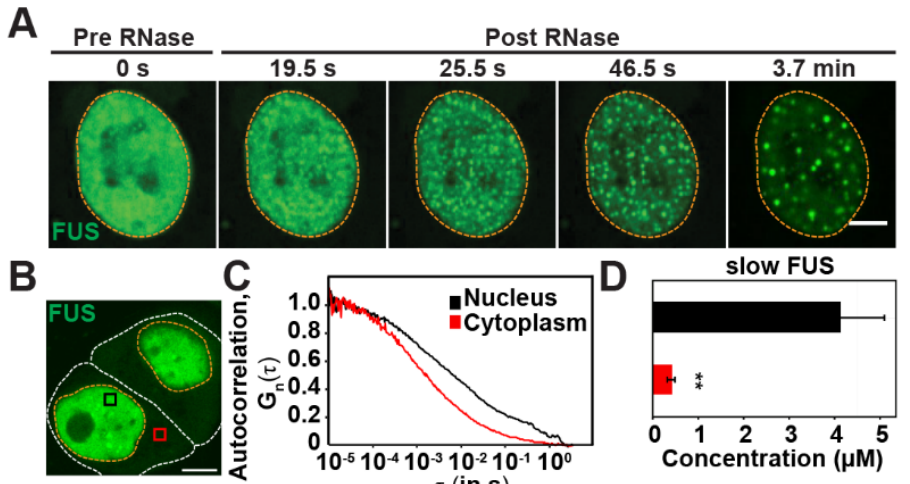

E
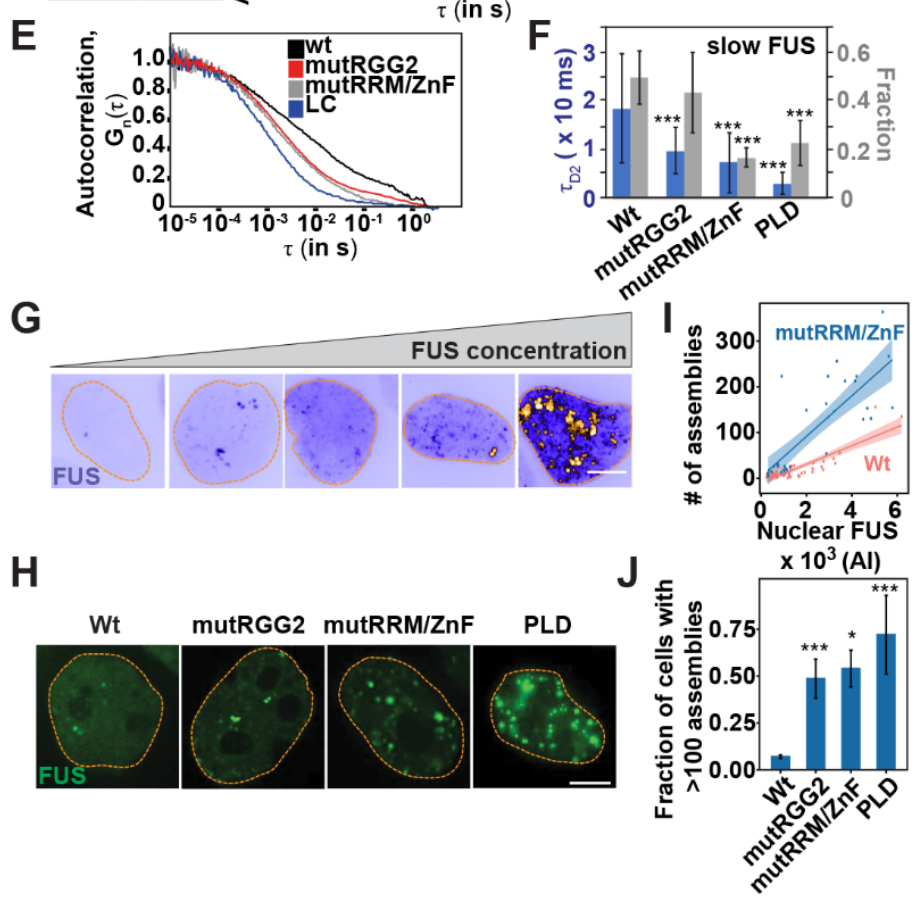

Fig. 3. RNA keeps prion-like RBPs in a soluble state in the nucleus. (A) Montage of a HeLa cell expressing FUSGFP after microinjection with RNase A. Scale bar, $2 \mu \mathrm{m}$. (B) HeLa cells expressing FUS-GFP. White lines indicate cell outlines, orange lines indicate nuclear outlines and boxes indicate regions of FCS measurements. Scale bar, $5 \mu \mathrm{m}$. (C) Autocorrelation curves obtained from FCS of FUS-GFP. (D) Quantification of the amount of slow FUS (see methods). Error bars represent SD. (E) Autocorrelation curves obtained from FCS of FUS-GFP variants in the nucleus. (F) Quantification of slow FUS in the nucleus, obtained from two component fits of the curves in $\mathrm{E}$ (see methods). Error bars represent SD. (G) HeLa cells showing variable FUS-GFP expression. (H) HeLa cells expressing different FUS-GFP variants with mutations in RNA binding domains (fig. S16). (I) Number of nuclear FUS-GFP assemblies per cell as a function of mean protein intensity (Al) $(n>30)$. (J) Number of cells with more than 100 nuclear assemblies, $n>100$ cells. Error bars represent SD. ${ }^{*} P<0.05$ and ${ }^{* *} P<0.01$ as compared to Wt. 


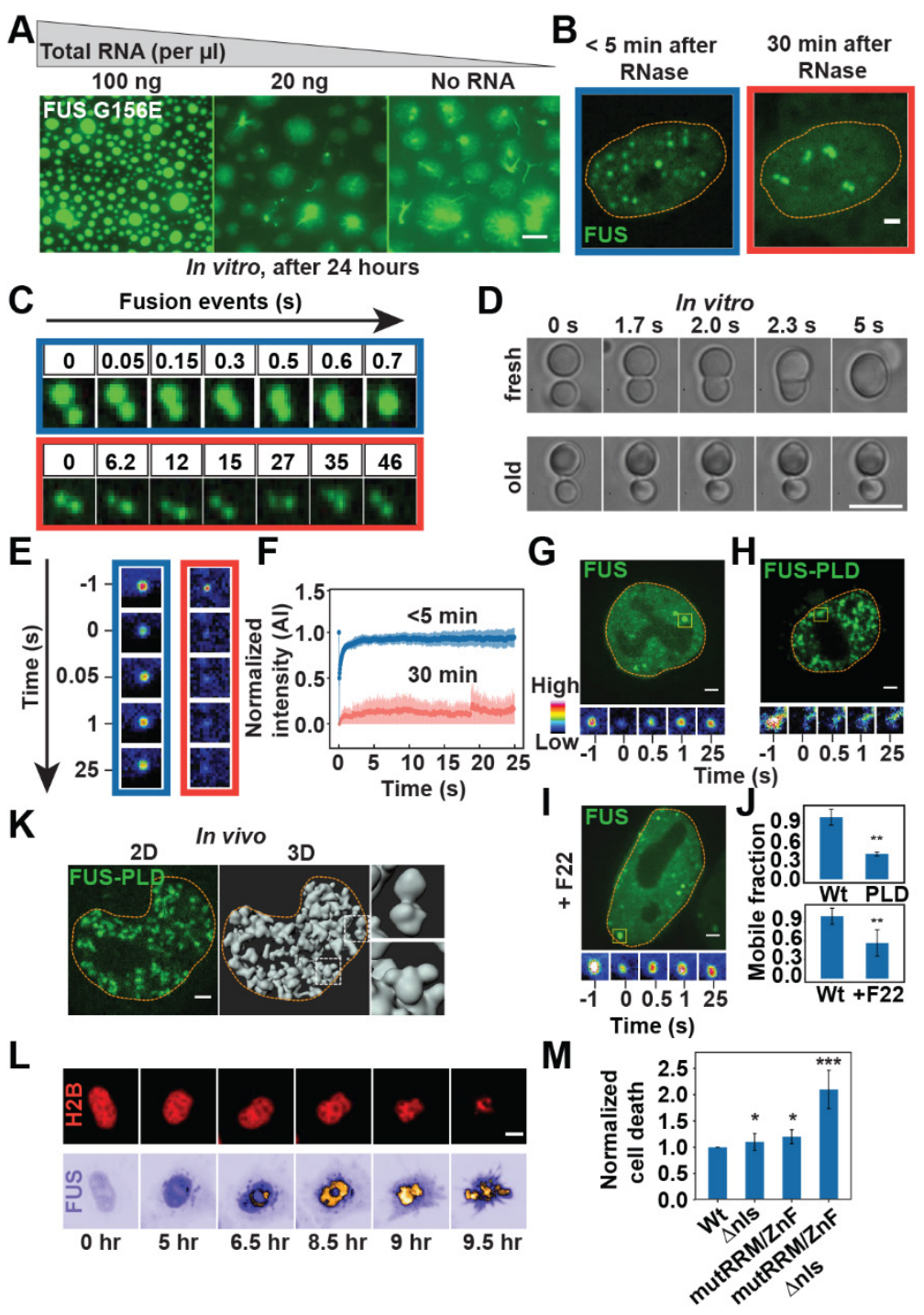

Fig. 4. RNA regulates aberrant liquid-to-solid phase transitions of prionlike RBPs. (A) In vitro phase separated FUS(G156E)-GFP in the absence or presence of total RNA after 24 hours. Scale bar, $2 \mu \mathrm{m}$. (B) FUS-GFP expressing HeLa cell nucleus after RNase A microinjection. (C) Montage of FUS-GFP droplets formed after RNase A microinjection. The droplets fuse in the first 5 min (blue box), but not after 30 min, resulting in "sticky droplets" (red box). (D) Montage of FUS-GFP droplets formed in vitro $(7 \mu \mathrm{M})$. Fusion of freshly formed droplets is compared to 3-hour old droplets. (E) Fluorescence recovery after photobleaching of nuclear FUS-GFP assemblies less than $5 \mathrm{~min}$ (blue box) or more than 30 min (red box) after RNase A microinjection. (F) FRAP of nuclear FUS-GFP assemblies in HeLa cells after RNA degradation as shown in $B$ and $E(n>10)$. ( $G$ to I) FRAP of nuclear assemblies in HeLa cells expressing full-length FUS $(\mathrm{G}, \mathrm{I})$ or FUS-PLD $(\mathrm{H})$. The cell in (I) was also treated with F22. $(J)$ Mobile fraction of photobleached assemblies in $G-I(n>15)$. Error bars represent SD. (K) 3D rendering of FUS-PLD nuclear assemblies. The inset shows aberrant "sticky droplets". Scale bar, $1 \mu \mathrm{m}$. (L) Time series to track the life time of FUS-GFP HeLa cells. H2B-mCherry was used to detect cell death. (M) Quantification of the fraction of cells undergoing cell death. 